www.jmscr.igmpublication.org

Impact Factor (SJIF): 6.379

Index Copernicus Value: 79.54

ISSN (e)-2347-176x ISSN (p) 2455-0450

crossrefDOI: https://dx.doi.org/10.18535/jmscr/v6i10.226

Journal Of Medical Science And Clinical Research

\title{
Role of serum Vitamin B12 and folic acid in Age related hearing loss
}

\author{
Authors \\ Dr Digant Patni (Asst. Prof.) ${ }^{1}$, Dr Maitri Kaushik (Prof. \& HOD) ${ }^{2}$ \\ Dr Arun Dehadaray (Prof.) ${ }^{3}$ \\ ${ }^{1}$ Dept. of ENT, Sri Aurobindo Medical College \& P.G. Institute, Indore \\ ${ }^{2,3}$ Dept. of ENT, Bharati Vidyapeeth Medical College, Pune \\ Corresponding Author \\ Dr Maitri Kaushik
}

\begin{abstract}
Introduction: Age related hearing loss (ARHL) is a common troublesome disorder of inner ear in elderly. It is characterized by degenerative changes in hair cells leading to slow, insidious, progressive hearing loss. Deficiency of multivitamin \& minerals are common in elderly and has been advocated in etiopathology of ARHL.
\end{abstract}

Aim \& Objective: To compare serum vitamin B12 \& folic acid in cases of age related hearing loss with normal population.

Materials \& Methods: In this prospective study through audiometric assessment was done of 80 patients of ARHL \& 80 normal subjects aged 40-60 years. All patients underwent blood estimation of Hemoglobin, BSL, Serum vitamin B12, Folic acid. Data analysis was done by SPSS software.

Results: It was found that serum folic acid was significantly low $(P=0.03)$ in ARHL group as compared to normal subjects. On the other hand, serum vitamin B12 levels showed no statistical difference among both ARHL and control group.

Conclusion: This study indicates low level of serum folic acid is associated with ARHL.

Keywords: Presbyacusis, Multivitamins, Vitamin B12, Folic acid, SNHL.

\section{Introduction}

Hearing loss is a common disorder in elderly. It is most prevalent human auditory disorder. Along with heart problems, hypertension \& arthritis hearing impairment is 1 of the 4 leading chronic health conditions experienced by the elderly ${ }^{(1)}$. Despite the high prevalence of hearing impairment, the biological basis of age-related hearing loss is unknown.

Several vitamin groups like vitamin B12, folic acid etc. has been identified as a possible etiological factors. Deficiency of micronutrients \& antioxidants cause oxidative stress \& free radical formation which results in inner ear damage (cochlear \& vestibular hair cells). Deficiency of these vitamins is common in elderly but more common vitamin inadequacy is of vitamin B12 \& folic acid ${ }^{(2,3)}$.

Low status of vitamin B12 \& folic acid / folate cause impaired methylation reaction in myelin synthesis. Due to deficiency of vitamin B12 \& folic acid there is increase in homocysteine levels 
which cause cholesterol deposition in arterial wall thus reducing blood flow, nutrients \& oxygen flow to the cochlea ${ }^{(4)}$

Only a few investigators have examined the possible associations of role of these nutrients in age-related hearing loss. Therefore, the objective of this study was to determine whether age-related hearing loss is associated with poor vitamin B-12 status, poor folate status, or both.

Study Design: Prospective Observational Study

\section{Materials \& Methods}

In this prospective study between 2011-2013, patients of age related hearing loss $(n=80) \&$ normal subjects $(n=80)$ with normal hearing were recruited in study between age group of 40-60 years. The patients who have the history of using ototoxic drugs, previous ear diseases, trauma to head \& the ear, \& familial history of hearing diseases were excluded from this study. Before clinical \& laboratory investigations, consent of all subjects were taken. Complete physical examination of ear, nose $\&$ throat and audiometric evaluation were performed in all subjects. Age related hearing loss (ARHL) is defined as mid to late adult onset, symmetrical, bilateral, progressive sensorineural hearing loss ${ }^{(5)}$.

The audiometric tests were performed in a soundproof chamber with and by Elkon eda $3 \mathrm{~N} 3$ diagnostic audiometer with head phones TDH 49 Bone vibrator - radio ear B71that was calibrated according to ISO standards. Hearing loss was calculated by taking an average of Air conduction threshold at octave interval of $0.5 \mathrm{KHz}, 1 \mathrm{KHz} \& 2$ $\mathrm{KHz}$ for flat type of audiogram which was called PTA1. In case of high frequency sloping type of audiogram hearing loss was calculated by taking average of Air conduction thresholds at octave interval of $1 \mathrm{KHz}, 2 \mathrm{KHz}, 4 \mathrm{KHz}$ and was called PTA2. Sensorineural type of hearing loss on PTA was accepted when Air-Bone gap was less than 10 $\mathrm{dB}$ (above normal hearing levels, when calculated in PTA 1 \& PTA 2). Normal hearing was accepted on PTA when hearing levels were within normal range $(0$ to $15 \mathrm{~dB})$ with air bone gap less than $10 \mathrm{~dB}^{(6)}$.

Blood samples were obtained from all subjects for determination of hemoglobin, random blood sugar levels. Anemia and diabetes mellitus patients were excluded. Further, Serum estimation of vitamin B12 \& folic acid was done. The blood samples were collected in plain bulb for estimation of serum vitamin B12 \& folic acid. Normal range of Vitamin B12 \& Folic acid according to same standard laboratory are $211-911 \mathrm{pg} / \mathrm{ml}$ and Above $5.38 \mathrm{ng} / \mathrm{dl}$, respectively.

Statistical analysis was done by using SPSS 17.0 statistical software. Two independent sample t-test, chi square test, Fisher's exact test to find the significance between ARHL group and control group with respect to various parameters.

\section{Results}

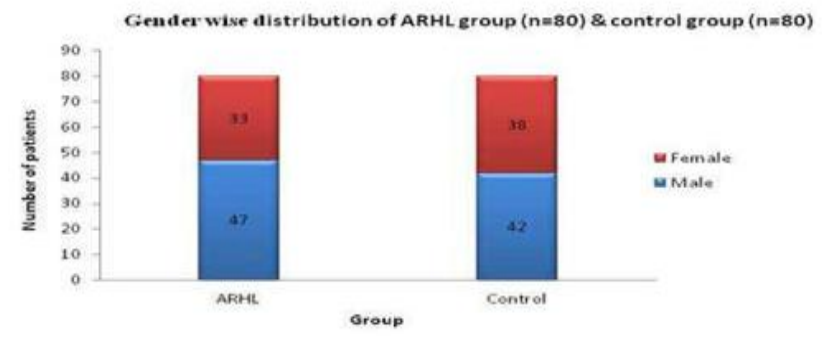

Figure: 01 Gender wise distribution of ARHL group $(n=80) \&$ control group $(n=80)$

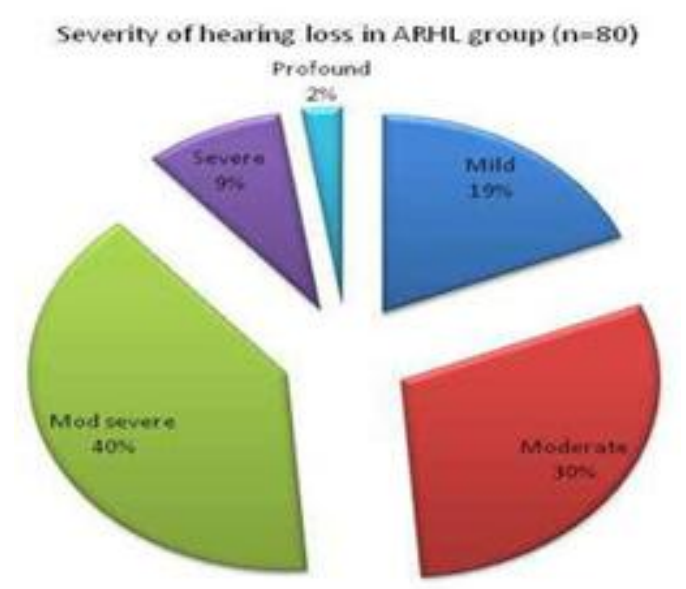

Figure: 02 Severity of hearing loss in ARHL group $(n=80)$ 


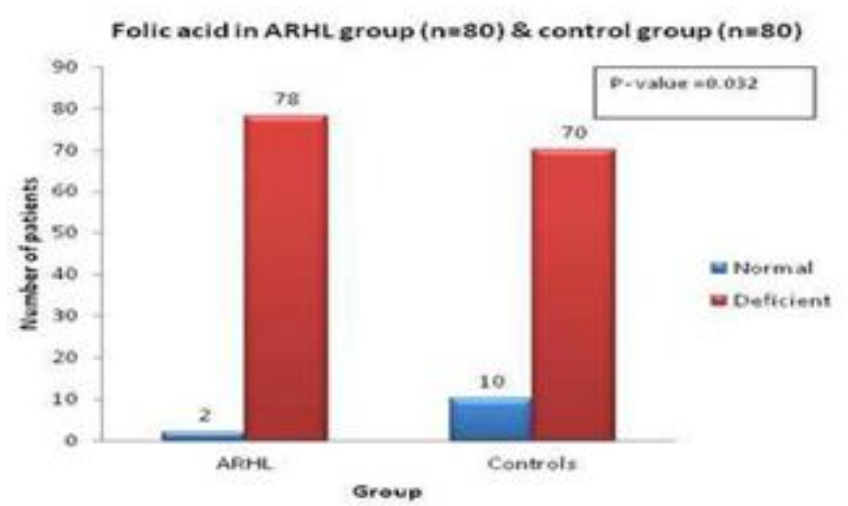

Figure: 03 Folic Acid in ARHL group $(\mathrm{n}=80) \&$ control $(n=80)$

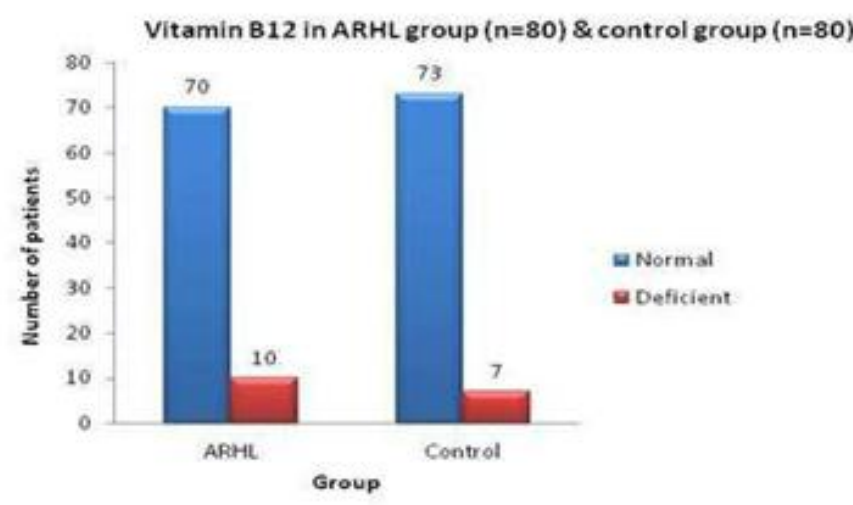

Figure: 04 Vitamin B12 in ARHL group $(n=80) \&$ control group $(n=80)$

Patients enrolled in study in ARHL group \& control group showed an even distribution (Fig. 1). On basis of pure tone audiometry, patients were divided on basis of severity of hearing loss (Fig 2) . In present study, majority ie $40 \%$ of patients were with moderately severe hearing loss followed by $30 \%$ with moderate SNHL. Serum Folic acid was deficient in $97.5 \%$ \& $87.5 \%$ patients in ARHL \& control group, respectively. There is a good correlation between serum folic acid deficiency \& ARHL with p-value being 0.032 which is statistically significant (Fig 3). Serum Vitamin B12 was deficient in $12.5 \% \& 8.75 \%$ of patients in ARHL \& control group, respectively. Thus showing a poor correlation between vitamin B12 \& ARHL with p-value being 0.609, which is statistically insignificant (Fig 4). Serum vitamin B12 and folic acid distribution in males and females of ARHL group and control group showed poor correlation which was statistically insignificant

\section{Discussion}

Deterioration in hearing thresholds is an expected outcome with aging. It is mainly due to loss of hair cells and other cellular elements of cochlea. Several factors like vascular, biochemical and genetic are proposed to play role auditory dysfunction $^{(7)}$. Multivitamins, antioxidants \& minerals are essential nerve regeneration, and myelin formation. Low status of vitamin B12 \& folic acid /folate cause impaired methylation reaction in myelin synthesis. Deficiencies of vitamin B-12 and folate or impaired methionine synthase activity results in decreased

S-adenosylmethionine synthesis, the primary source of methyl groups in the nervous system, which may impair methylation reactions in myelin synthesis. Also due to deficiency of vitamin B12 \& folic acid there is increase in homocysteine levels which cause cholesterol deposition in arterial wall thus reducing blood flow, nutrients, and oxygen to different organs including ears i.e. reduce blood flow to the cochlea ${ }^{(8,9)}$. The apex and base of the cochlea are responsible for transducing lowfrequency sounds and high-frequency sounds, respectively. It has been postulated that the cochlear sensory cells of the apex may be the most susceptible to aberrations in microcirculation in the stria vascularis because the apex is the farthest from the blood supply of the cochlea ${ }^{(10)}$. Finally, age related hearing loss initially affects high frequency hearing and slowly extends to hearing in the lower frequencies; hence corresponding sensory loss occurs earlier in the high frequencies than in lower frequencies.

In the present study, low levels of serum folic acid was found more in ARHL goup as compared to control group with p-value being 0.032. Celil $\mathrm{G}$ et $\mathrm{al}^{(7)}$, found, significantly lower levels of serum folate was associated with ARHL $(\mathrm{p}=0.029)$. Houstan et $\mathrm{al}^{(2)}$, concluded, women with ARHL had lower serum folic acid and vitamin B12. They reported poor folate and vitamin B12 status may be associated with ARHL. Contrary to this study, like Berner et $\mathrm{al}^{(11)}$, Gopinath $\mathrm{B}^{(9)}$ et al showed poor correlation between status of folic acid and ARHL. 
Though work has been done regarding influence of folate deficiency on auditory function but has received little attention.

Several investigators have examined the association between vitamin B-12 status and auditory function (12-17). In our study, there was a poor correlation between status of serum vitamin B12 \& ARHL, with p-value being 0.609. Berner et $\mathrm{al}^{(11)}$, Gopinath $\mathrm{B}$ et $\mathrm{al}^{(9)}$ and Shargorodsky et $\mathrm{al}^{(18)}$ also showed statistically inginificant correlation between vitamin B12 and ARHL. However, there is little evidence in the literature that age-related hearing loss is associated with vitamin B-12 deficiency. Vitamin B-12 deficiency and auditory dysfunction have been linked primarily in studies of neuropathology, tinnitus, and abnormal auditory brainstem response ${ }^{(16,17)}$. Shemesh et $\mathrm{al}^{(13)}$ examined serum vitamin B-12 in 113 subjects with chronic tinnitus and noiseinduced hearing loss. They found that it is likely to be vitamin B-12-deficient than subjects with normal hearing. Shemesh et $\mathrm{al}^{(13)}$ suggested that inadequate vitamin B-12 may be associated with myelin damage in persons with repeated noise exposure. Some subjective improvement in tinnitus was observed in 12 subjects after vitamin B-12 replacement therapy.

Our sample size was relatively small and consisted of ARHL group of hearing loss patients and control group with healthy individuals aged 40-60 years. Thus, additional studies are needed to determine the roles of vitamin B-12 and folate in auditory dysfunction.

Additional research is needed to confirm findings of present study and to determine whether, multivitamins status especially poor serum vitamin B-12 and folate pose modifiable risk factors for age-related hearing impairment.

\section{Conclusion}

Deficiency of folic acid in a ARHL was found to be significant as compared to normal population. Folic acid deficiency in elderly has a significant role in age related hearing loss as it could affect the function of inner hair cells at molecular levels.
Low levels of multivitamins especially serum vitamin B12 \& folic acid could be an indicator of early ARHL which is a modifiable risk factor. However, further large scale study is needed to substantiate it.

\section{References}

1. Bureau of the Census, US Department of Commerce, Economics and Statistics Administration. Statistical Abstract of the United States, 114th ed. Washington, DC: US Government Printing Office, 1994.

2. Houston DK, Johnson MA, Nozza RJ, Gunter EW, Shea KJ, Cutler GM, et al. Age-related hearing loss, vitamin B-12, and folate in elderly women. Am J Clin Nutr. 1999;69:56471. [PMID: 10075346]

3. Andres R, Hallfrisch J. Nutrient intake recommendations needed for the older American. J Am Diet Assoc 1989;89:1739-41.

4. Ubbink JB, Vermaak WJH, Van der Merwe A , Becker PJ. VitaminB12, vitamin B6, and folate nutritional status in men with hyperhomocysteinemia .Am J Clin Nutr 1993; 57:47-53.

5. David M Baguley, Evan Reid, Andrew McCombe .Age related sensorineural hearing loss. Scott-Brown's Otolarhinolaryngology, Head and Neck Surgery 2008; Hodder Arnold $7^{\text {th }}$ edition; 2(238a) :3539-47

6. Stanely A Gelfend. Essentials of Audiology. $2^{\text {nd }}$ edn. New York: Thieme ;2001

7. Celil Gocer, Umut Genc, Adil Eryilmaz, Ahmet Islam, Suleyman Boynuegri, Fatih Bakir. Homocysteine, Folate and Votamin B12 concentration in Middle Aged Adults Presenting with Sensorineural Hearing Impairment. Int Adv Otol. 2009;5(3) 340-344.

8. Savage DG, Lindenbaum J. Folate-cobalamin interactions. In: Bailey LB, ed. Folate in health and disease. New York: Marcel Dekker, 1995:237-86.

9. Bamini Gopinath, Victoria Flood, Elena Rochtchina, Catherine McMahon, Paul Mitchell. Serum Homocysteine and Folate 
concentration are associated with prevalent Age related hearing loss.J.Nutr.2010;140:146974

10. Gates GA, Cobb JL, D'Agostino RB, Wolf PA. The relation of hearing in the elderly to the presence of cardiovascular disease and cardiovascular risk actors. Arch Otolaryngol Head Neck Surg. 1993;119:156-61.

11. Berner B, Odum L, Parving A. Age-Related Hearing Impairment and B Vitamin Status. Acta Otolaryngol 2000; 120: 633-7

12. Roman GC. An epidemic in Cuba of optic neuropathy, sensorineural deafness, peripheral sensory neuropathy and dorsolateral myeloneuropathy. J Neurol Sci 1994;127:11-28

13. Shemesh Z, Attias J, Ornan M, Shapira N, Shahar A. Vitamin B12 deficiency in patients with chronic-tinnitus and noise-induced hearing loss. Am J Otolaryngol 1993;14:94-9.

14. Agamanolis DP, Chester EM, Victor M, Kark JA, Hines JD, Harris JW. Neuropathology of experimental vitamin B12 deficiency in monkeys. Neurology 1976;26:905-14.

15. Krumholz A, Weiss H, Goldstein P, Harris K. Evoked responses invitamin B12 deficiency. Ann Neurol 1981;9:407-9.

16. Fine E, Hallett M. Neurophysiological study of subacute combined degeneration. J Neurol Sci 1980;45:331-6.

17. Fine E, Soria E, Paroski M, Petryk D, Thomasula L. The neurophysiologicalprofile of vitamin B12 deficiency. Muscle Nerve 1990;13:158-64.

18. Josef Shargorodsky, Sharon Curhan, Roland Eavey, Gary Curhan. A prospective study of vitamin intake and the risk of hearing loss in men. Otolaryngol Head Neck Surg 2010 Feb;142(2):231-6. 